\title{
GENES FOR REPRODUCTIVE ISOLATION LOCATED ON REARRANGED CHROMOSOMES
}

\author{
Y. SANO and F. KITA \\ National Institute of Genetics, Misima, 411, and Faculty of Agriculture, \\ Hokkaido University, Sapporo, 060, Japan
}

Received 8.iv.78

\section{SUMMARY}

\begin{abstract}
Evidence from an experiment involving interspecific hybrids of Melilotus suggested that several genes causing isolating barriers, such as hybrid breakdown and non-crossability, are located on rearranged segments. In general, reproductive barriers were controlled by complementary or duplicate genes. The fixation of such genes within a population is possibly promoted when they are linked with the breakage points of rearrangements.
\end{abstract}

\section{INTRODUCTION}

THERE are two hypotheses proposed for the functional role of rearranged segments between species though our knowledge about the genic contents on rearranged chromosomes is quite limited. The first is that the chromosomal rearrangenents or sterility due to heterozygosity for translocation and inversion of small chromosomal segments are inherited independently of the morphological and physiological differences between the parental species (Stebbins, 1950). The second is that rearranged segments normally contain genes affecting growth and vigour and/or genes determining morphological differences between the parental species (Grant, 1956, 1966, 1967). It is not easy to resolve the discrepancy because hybrid sterility or distorted segregation frequently observed in the interspecific crosses makes the result obscure.

Supporting the latter hypothesis, three lines of evidence were obtained from the present experiment on Melilotus which suggested that several genes causing isolating barriers are located on the interchanged segments, and as a result, simultaneous fixation of different factors for isolating mechanisms might promote speciation reducing substitutional genetic load.

All Melilotus species (sweetclover) have the same chromosome number and are diploid $(2 n=16)$ (Smith and Gorz, 1965). Reproductive barriers such as crossing barriers and hybrid inviability have been reported from interspecific cross experiments (Greenshields, 1954; Smith, 1954). In addition, $M$. alba differs from the three species, $M$. officinalis, $M$. polonica and $M$. suaveolens by a reciprocal translocation (interchange), while the latter three species have the same chromosomal constitution and no disturbance in chromosomal pairing at meiosis was detected in their $F_{1}$ hybrids (Sano and Kita, 1975). Among these four species, hybrid sterility observed in $F_{1}$ and $F_{2}$ generations was mainly caused by interchange. Accordingly, it was possible to carry out a standard factorial analysis. The effects of both genic and chromosomal segregations are reported in the present paper and the evolutionary significance of genic differentiation on rearranged segments is discussed. 


\section{Materials and methods}

Eight strains belonging to four species of the genus Melilotus, M. alba, $M$. officinalis, $M$. polonica and $M$. suaveolens, were used in the study. The seeds were available through the courtesy of H. J. Gorz (U.S.A.) and G. A. Stevenson (Canada).

Meiotic configurations at diakinesis and first metaphase were analysed to determine whether the plant was homozygous or heterozygous for interchange. In the $\mathrm{F}_{3}$ of $M$. officinalis $\times M$. alba, the decision whether or not a plant is homozygous for interchange could be made on the basis of pollen fertility since in the $F_{2}$ and $B_{1} F_{2}$ of the cross, pollen infertility was mainly caused by interchange. In the other cases, chromosomal pairing was examined in each of the plants.

\section{Results}

\section{(i) Chlorosis gene}

$\mathrm{F}_{1}$ plants between $M$. alba and $M$. suaveolens were heterozygous for an interchange (Sano and Kita, 1975). Although the $F_{1}$ was normally green in leaf colour, chlorotic segregants occurred in the $F_{2}$ population with a frequency of about $1 / 8$, due to the segregation of two sets of duplicate genes, $c h l_{1}$ and $c h l_{2}$ for lethal type and $c h w_{1}$ and $c h w_{2}$ for weak type (Sano and $\mathrm{Kita}, 1978$ ). As double recessive genotypes are considered to be chlorotic, each of the lethal and weak types has a frequency of $1 / 16$ in $\mathrm{F}_{2}$. The $c h l_{2}$ and $c h w_{2}$ genes which are carried by $M$. suaveolens were linked with a recombination value of about 13 per cent. The backcross data of $M$. alba Arctic $\times$ (M. alba Arctic $\times M$. suaveolens 18-4) were reanalysed to detect linkage relationships between the breakage point and $c h l_{2}$ or $c h w_{2}$. For simplicity, only a case of lethality is illustrated in fig. 1 . In this case, $c h l_{2}$ is linked with the breakage point but $c h l_{1}$ is independent. The frequency of chlorotic segregants is $1 / 4$ or $1 / 16$ in segregating lines of $B_{1} F_{2}$. Taking chromosomal segregation into account, four types of segregation ( $a, b, c$ and $d$ in fig. l) are expected to occur. Then, the recombination value $(p)$ between the breakage point and $c h l_{2}$ can be obtained by $P=\frac{c+d}{n} \pm \sqrt{\frac{p(1-p)}{n}}$, where $n$ is total number of lines observed.

In fact, the genotype of $M$. alba was $c h l_{1} c h w_{1}+_{2} t_{2}$ and that of $M$. suavelolens was $+_{1} t_{1} c h l_{2} c h w_{2}$. The ratio of fixed normal to segregating lines $(a+d: b+c)$ for the lethal type, $12: 11$, and that for the weak type,

\section{TABLE 1}

Segregation for interchange and chlorosis genes in the backcross of M. alba $\times$ (M. alba $\times$ M. suaveolens)

Types of

segregation

Non-cross-over type

a

$\mathrm{b}$

Cross-over type

c

d
No. of lines observed

$\begin{array}{cr}\text { Lethality } & \text { Weakness } \\ 9 & 9 \\ 9 & 8 \\ & \\ 2 & 2 \\ 3 & 4\end{array}$

Types of segregation are represented in fig. 1 . 


$$
\begin{array}{llll}
\text { Parents } \quad F_{1} \text { hybrid Gametes } & \begin{array}{l}
\text { Inter- Chlorosis Types of } \\
\text { change }
\end{array}\left(\mathrm{B}_{1} \mathrm{~F}_{2}\right) \text { segregation }
\end{array}
$$

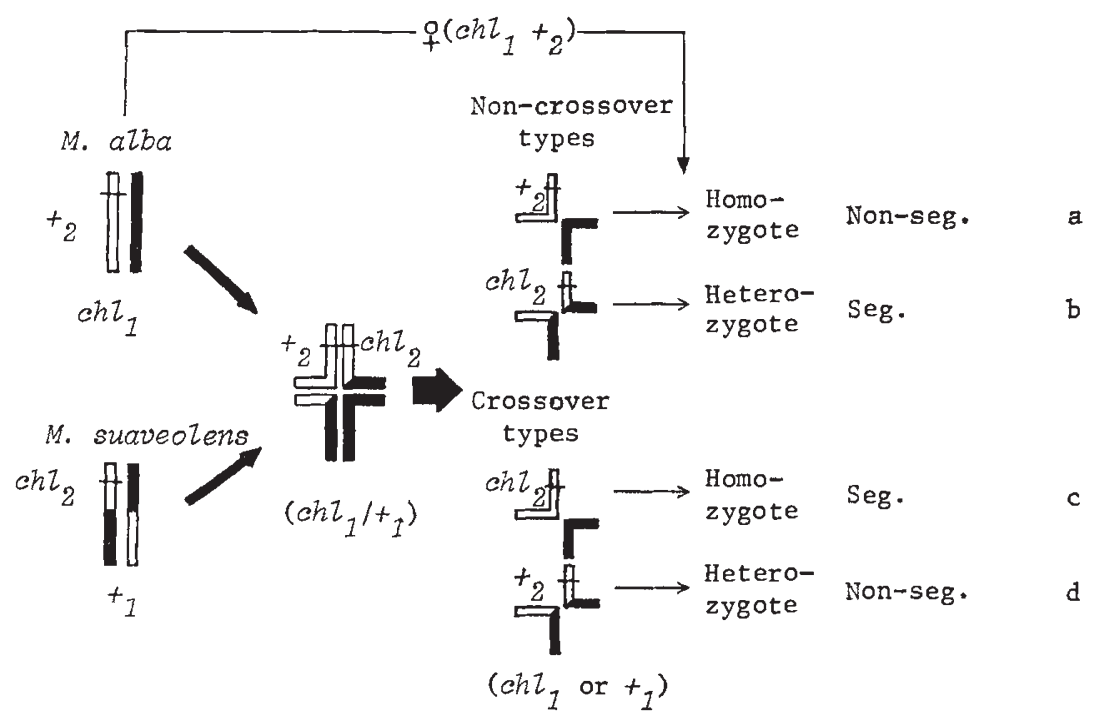

FIG. 1.-Four types of segregation for interchange and chlorosis in the backcross of M. alba $\times$ (M. alba $\times$ M. suaveolens).

$13: 10$, both gave a good fit to the expected $1: 1$ ratio (table 1 ) supporting the assumption that two sets of duplicate genes exist. Meiosis was examined in each of the $B_{1} F_{1}$ plants to detect whether or not it had a quadrivalent or a trivalent. The observed ratio of interchange heterozygotes to homozygotes $(b+d: a+c, 12: 11)$ also agrees with a $1: 1$ ratio. $\chi^{2}$ for linkage between the breakage point and $c h l_{2}$ or $c h w_{2}$ was $7.425(P<0.01$, d.f. $=1)$ or 5.490 $(P<0.05$, d.f. $=1)$. The estimated recombination values were $0.217 \pm 0.086$ and $0 \cdot 261 \pm 0 \cdot 092$, respectively. The linkage relationships are indicated as follows:

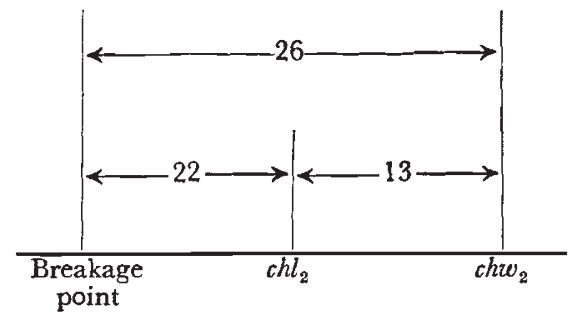

$\mathrm{F}_{1}$ hybrids of $M$. officinalis P.I. $178985 \times M$. alba Bdn 928 or Brandon Dwarf were also heterozygous for interchange and had as high seed fertility (about 65 per cent) as the mean of the parents. Chlorotic and inviable segregants occurred with high frequencies from 60 to 70 per cent in the $F_{2}$ generation. Out of 182 plants which survived in the $F_{2}$ generation 151 proved to have a quadrivalent or a trivalent at diakinesis or first metaphase in the PMCs, which showed that they were heterozygous for the interchange, while the number of homozygotes was 28 and the remaining three were aneuploid. 
An observed ratio of heterozygotes $v$. homozygotes for interchange was significantly distorted from the expected ratio of $1: 1 \quad\left(\chi^{2}=84.520\right.$, $P<0.001$, d.f. $=1$ ).

To investigate how interchange homozygotes were eliminated, correlation coefficients were computed between the frequency of heterozygotes in $\mathrm{F}_{3}$ lines, $F_{2}$ pollen fertility, $F_{2}$ seed fertility and mortality due to chlorosis in $F_{3}$ lines. As shown in fig. 2, the frequent occurrence of heterozygotes was positively correlated with mortality due to chlorosis $(r=0.659$, d.f. $=26$,

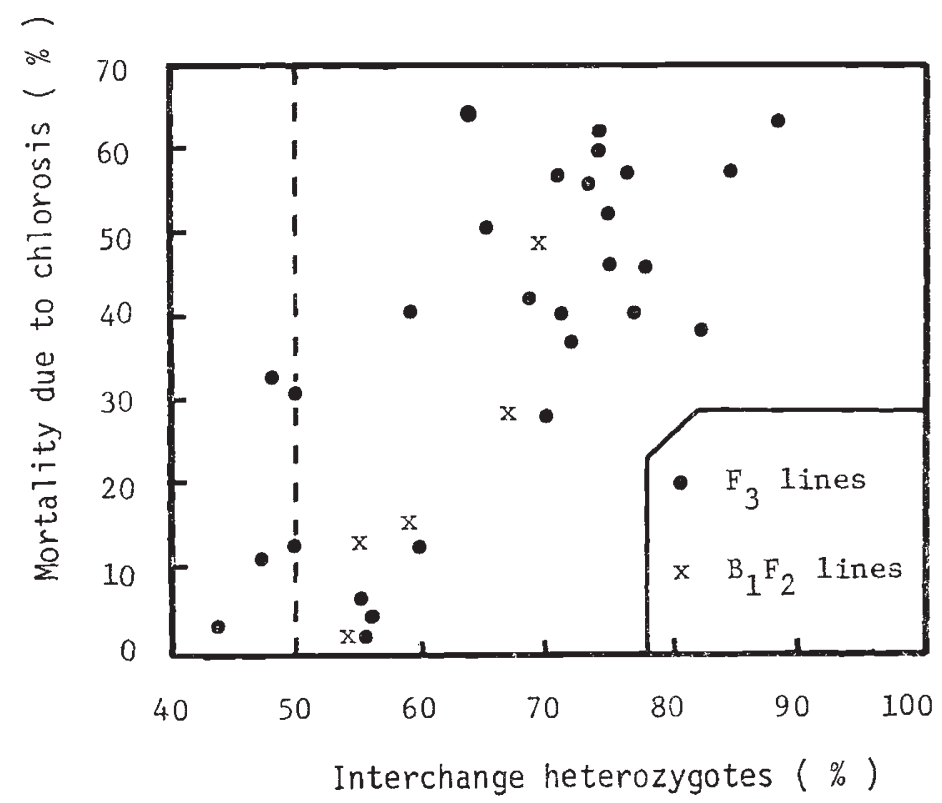

FIG. 2.- Relationship between mortality due to chlorosis and frequency of interchange heterozygotes in the cross between $M$. officinalis and $M$. alba. Mean numbers of plants per line which survived in $\mathrm{F}_{3}$ and $\mathrm{B}_{1} \mathrm{~F}_{2}$ are $20 \cdot 3$ and $35 \cdot 6$, respectively.

significant at 1 per cent level). In five $\mathrm{B}_{1} \mathrm{~F}_{2}$ lines segregating for interchange, lines with a high frequency of heterozygotes also seemed to result from high mortality due to chlorosis (fig. 2). It should be noted that in the case where mortality due to chlorosis is low the ratio heterozygotes $v$. homozygotes for interchange tends to be the expected ratio of $1: 1$. Chlorosis in this case may also be controlled by many sets of duplicate or complementary recessive genes since $F_{1}$ and $B_{1}$ plants were always viable here as well as in the cross of $M$. alba and $M$. suaveolens. Several genes causing hybrid breakdown seem to be linked with the breakage point of interchange, which could also bring about elimination of homozygotes.

\section{(ii) Crossability gene}

The third line of evidence that genes causing isolating barriers are located on rearranged segments was obtained from the three-species cross of $M$. polonica $\times(M$. alba $\times M$. suaveolens $)$. $M$. alba was crossable with both $M$. polonica and $M$. suaveolens though the latter two species could not be 
crossed when a strain of $M$. polonica, 3446, was used. The cross-fertility of M. alba $\times M$. suaveolens was 23 per cent; that of $M$. polonica $\times M$. alba was 28 per cent. When $M$. polonica was pollinated with the pollen of the albasuaveolens $\mathrm{F}_{1}$ hybrid, the cross-fertility was reduced to about a half of the above values (about 13 per cent), and strongly shrunken seeds were obtained in approximately the same proportion $(53: 64)$. A $1: 1$ ratio of normal $v$. shrunken seeds suggests that crossability is controlled by a few major and complementary dominant genes.

In addition, the three-species cross is expected to give a ratio of one heterozygote to one homozygote for interchange unless both types of chromosomal constitution are eliminated through abortion of the young embryo, since both of them segregated in a $1: 1$ ratio in pollen of $M$. alba $\times M$. suaveolens $\mathrm{F}_{1}$ (table 1). The number of interchange homozygotes was, however, observed to be significantly less than that of heterozygotes (table 2).

TABLE 2

Segregation in the three-species cross of M. polonica $\times($ M. alba $\times$ M. suaveolens)

\begin{tabular}{|c|c|c|c|c|c|c|}
\hline \multirow[b]{2}{*}{$\begin{array}{l}\text { Locus } \\
\text { Breakage point }\end{array}$} & \multirow[b]{2}{*}{$\begin{array}{c}\text { Cross } \\
\text { A } \\
\text { B }\end{array}$} & \multicolumn{3}{|c|}{ No. of plants } & \multicolumn{2}{|c|}{ Goodness of fit } \\
\hline & & $\begin{array}{l}\text { Heterozygote } \\
29 \\
17\end{array}$ & $\begin{array}{c}\text { Homozygote } \\
15 \\
0\end{array}$ & $\begin{array}{c}\text { Total } \\
44 \\
17\end{array}$ & $\begin{array}{c}\chi^{2}(1: 1) \\
4.455 \\
17 \cdot 000\end{array}$ & $\begin{array}{c}\quad \mathbf{P} \\
<0.05 \\
<0.01\end{array}$ \\
\hline$B p$ & A & 23 & 21 & 44 & 0.091 & $0.90-0.75$ \\
\hline Ms & $\mathbf{A}$ & 19 & 25 & 44 & 0.818 & $0.50-0.30$ \\
\hline
\end{tabular}

Cross A: $M$. polonica $3446 \times(M$. alba Arctic $\times M$. suaveolens $18-4)$.

Cross B: M. polonica $3446 \times$ (M. alba Denta $\times M$. suaveolens Redfield Yellow).

As $M$. polonica has the same chromosomal constitution as $M$. suaveolens, this means that chromosomes of $M$. suaveolens tend to be eliminated. Then, the genes determining crossability between $M$. polonica and $M$. suaveolens appear to be located on interchanged segments. $M$. suaveolens has two dominant genes, $B p$ (black pod) and $M s$ (mottled seed), but the other species have neither. Each of them appeared to be independent of the breakage point from the result of backcrosses between $M$. alba and $M$. suaveolens. In the three-species cross, segregation for each of them showed a ratio of $1: 1$ as expected.

\section{Discussion}

There have been many discussions of the significant roles that isolating barriers and chromosomal changes play in speciation (Stebbins, 1950; Dobzhansky, 1951). When such variations exist within a population, they reduce the fitness of the population due to the occurrence of lethal and infertile individuals. Then, substitutional genetic load (Haldane, 1957) would be greater if such alteration takes place in speciation. The fact that among species much genic and chromosomal differentiation prevail might suggest the existence of mechanisms reducing genetic load.

On the other hand, it has been suggested that chromosomal polymorphisms are preserved in a population because of the adaptive superiority of the heterozygotes, this compensating for the sterility (Cleland, 1962; Bailey et al., 1976). This heterotic effect was explained by the heterozygous combinations of gene complexes located on the chromosomes involved in 
rearrangements. This explanation has a serious problem when such rearrangements with heterotic effects have to be fixed within a population, following differentiation in chromosomal constitution-both homozygotes to be fixed have selective disadvantages relative to the heterozygotes.

In general, reproductive barriers are controlled by complementary or duplicate genes (Sachs, 1953; Oka, 1957, 1974; Tsunewaki and Kihara, 1962; Moore, 1966; Chu and Oka, 1972; Takahashi et al., 1976). It has been suggested by Dobzhansky (1951) that one of the complementary genes could be selectively neutral and the most effective isolating mechanism could be produced through complementary action of genes. As shown in this paper, the fixation of certain combinations of them in each species gives rise to effective isolating barriers. Their fixation within a population is possibly promoted when such genes are linked with the breakage point of rearrangements. Linkage relations between genes for reproductive isolation and rearranged segments might offer an internal mechanism reducing substitutional genetic load and allowing a rapid speciation because deleterious factors tend to be selected against simultaneously. Further, the existence of such genes on rearranged segments might suggest reorganisation of genic contents on the segments at an early stage of speciation.

Acknowledgements.-We wish to express our hearty thanks to Professor M. E. Takahashi, Faculty of Agriculture, Hokkaido University, and Dr H. I. Oka, National Institute of Genetics, Japan, for their suggestions and discussions. We are also indebted to Dr H. J. Gorz, Crops Research Division, ARS, USDA, and Dr G. A. Stevenson, Canada Department of Agriculture, Manitoba, for their kind supply of stock seeds.

\section{REFERENCES}

BAILEY, R. J., REES, H., AND JONES, L. M. 1976. Interchange heterozygotes versus homozygotes. Heredity, 37, 109-112.

CHU, Y. E., AND OKA, H. I. 1972. The distribution and effects of genes causing $\mathrm{F}_{1}$ weakness in Oryza breviligulata and 0 . glaberrima. Genetics, 70, 163-173.

Cleland, R. E. 1962. The cytogenetics of Oenothera. Adv. Genet., 11, 147-238.

DoBzhansky, тh. 1951. Genetics and the Origin of Species, 3rd ed. Columbia Univ. Press, New York.

GRANT, v. 1956. Chromosome repatterning and adaptation. Adv. Genet., 8, 89-107.

GRANT, v. 1966. Block inheritance of viability genes in plant species. Amer. Nat., 100, 591-601.

GRANT, v. 1967. Linkage between morphology and viability in plant species. Amer. Nat., $101,125-139$.

GREENSHIELDS, J. E. R. 1954. Embryology of interspecific crosses in Melilotus. Can. F. Bot., $32,447-465$.

haldane, J. B. s. 1957. The cost of natural selection. F. Genet., 55, 511-524.

MOORE, K. 1966. The physiological control of $\mathrm{F}_{1}$ grass-dwarfs in Triticum aestivum $\mathrm{L}$. Euphytica, 15, 329-347.

OKA, H. I. 1957. Phylogenetic differentiation of cultivated rice. XV. Complementary lethal genes in rice. Fap. F. Genet., 32, 83-87.

OKA, H. I. 1974. Analysis of genes controlling $\mathrm{F}_{1}$ sterility in rice by the use of isogenic lines. Genetics, 77, 521-534.

SACHS, L. 1953. The occurrence of hybrid semi-lethals and the cytology of Triticum macha and T. vavilovi. F. Agric. Sci., 43, 204-213.

SANO, Y., AND KITA, F. 1975. Cytological studies of several interspecific hybrids in the subgenus Eumelilotus. 7. Fac. Agric., Hokkaido Univ., Sapporo, 58, 225-246.

SANo, Y., AND KITA, F. 1978. Reproductive barriers distributed in Melilotus species and their genetic bases. Can. 7. Genet. Cytol., 20, 275-289.

smrth, w. K. 1954. Viability of interspecific hybrids in Melilotus. Genetics, 39, 266-279. smrth, w. x., AND GoRz, H. J. 1965. Sweetclover improvement. Adv. Agron., 17, 164-231. 


\section{GENES FOR ISOLATION ON REARRANGED CHROMOSOMES 383}

STEBbins, G. I. 1950. Variation and Evolution in Plants. Columbia Univ. Press, New York. TAKAHASHI, R., HAYASHI, J., AND MORIYA, 1. 1976. Inheritance and linkage studies of the chlorotic hybrids due to complementary dominant genes. Barley Genet. Newslett., 6, 71-73.

TSUNEWAKI, K., AND KIHARA, H. 1962. Comparative gene analysis of common wheat and its ancestral species. I. Necrosis. Jap. 7. Genet., 37, 474-484. 